\title{
Mathematical Formula Computing Based on JavaEE and MATLAB
}

\author{
Haijun $\mathrm{Cao}^{1}$ and Meihua $\mathrm{Shi}^{2, *}$ \\ ${ }^{I}$ School of Science, Shandong Jiaotong University, ${ }^{2}$ Zhejiang International Studies University, China
}

\begin{abstract}
This article describes a method of the implementation of mathematical formula computing software system, which is a B/S structure system using Java EE technology. By this system, web user can get the result of a mathematical formula in case of inputting the Latex format.
\end{abstract}

Keywords: Mathematical formula, program, web page, LaTex format.

\section{INTRODUCTION}

Since the first computer was invented, the computer technology has been in a leap-type momentum on development, which has been applied widely in all fields such as production, living, scientific research, etc. the high-speed development of the computer technology has boosted the mathematical development greatly. However, it is still relatively tough for common users to calculate the mathematical formula by computers. Therefore, a large number of users have no way to operate computers in case of calculating the mathematical formula. In this case, it is necessary to develop calculating software for the mathematical formula (all the concepts and formulas can be found in [1-3]) based on network. In this way, users will be able to make the calculation by the software online.

\section{THE SELECTION OF THE APPLICABLE TECH- NOLOGY}

Currently, the mainstream technologies based on the software development of the WEB are ASP technology, PHP technology and JSP technology

(All the concepts and formulas can be find in [4-6]). We will formulate WEB utility software of MVC mode, applying the JSP technology and combining the framework of Struts2.x, Spring2.x (by [7]).

The advantage of the MVC mode lies in the hierarchical structure of the software, ie. it has divided the whole software into Model, View, and Controller. Strut is open software, which aims to help us reduce the time when applying the MVC design mode to develop the Web. Spring is open framework, which is to be created for the application and development of the enterprises.

Spring is a lightweight framework, which uses the basic Java Bean to complete the tasks which could only be done by the EJB. Spring specializes in all layer of the solution for J2EE, but not just in a certain layer. The application of Spring almost integrates all the current framework perfectly.

Now, the general computer users apply the software such as Word and Latex to input the mathematical formula. After having input the mathematical formula, what users will see is the printed style. However, the desired effect will be attained after the compilation in Latex. We herein choose the format of the mathematical formula, which is the one that can be recognized by the software system.

Because the input format of Latex will not be seen intuitively by users' eyes, but it is very easy and intuitive for computer to identify. Moreover, the application of the input format of Latex as the input format of the mathematical formula in the software can bring convenience for users to input the formula by keyboard in the webpage, which is the failure for Word format.

MATLAB (all the concepts and formulas can be found in $[2,3])$ in numerical calculation is a much needed tool. It is a collection that contains a large number of calculation algorithms, which has more than 600 mathematical functions so as to meet easily various computing needs from users.

\section{SYSTEM DESIGN}

We have to design application system which is based on the model of MVC and apply the technology of Java EE, meanwhile, it takes the numerical calculations by means of MATLAB.

\subsection{The Overview Design}

The system is to achieve the result after accepting the input of the formula. In this way, the system just formulates a webpage of JSP as well as a formula which is to receive, calculate and count the users' input and for users to input the formula without applying database and the background management system, and retrieve the result back from the business logic of the webpage of JSP. It can be seen from Fig. (1). 


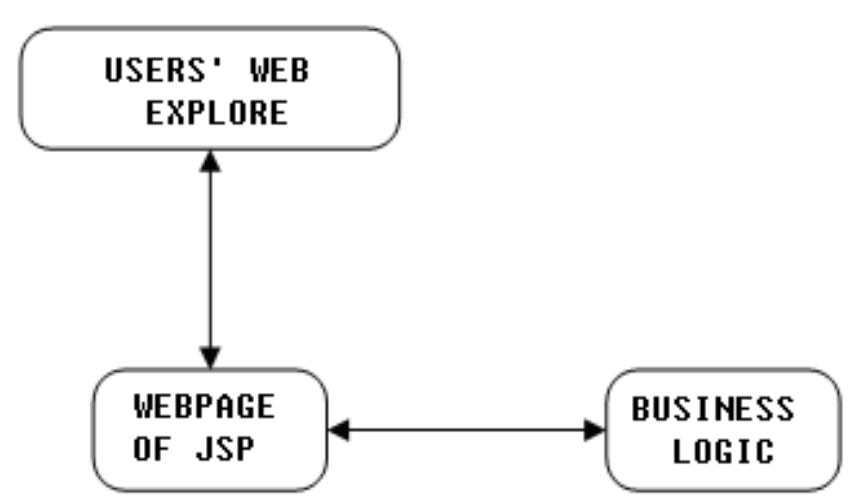

Fig. (1). Logic of the webpage of JSP.

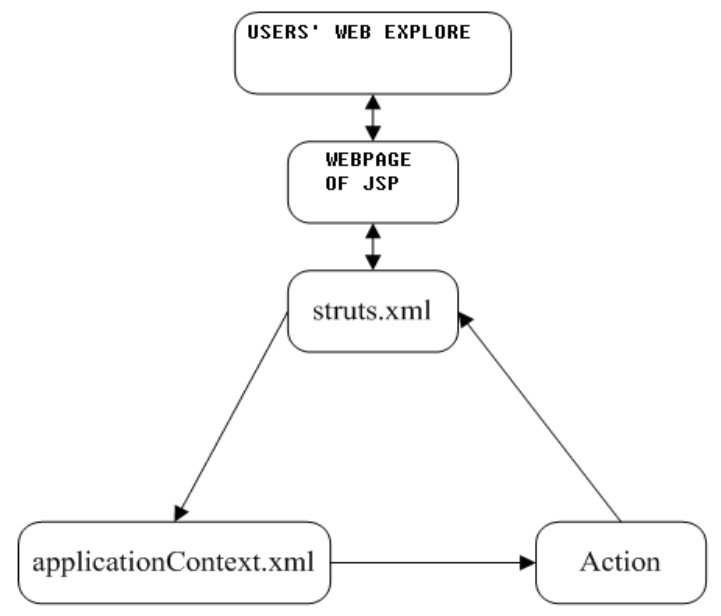

Fig. (2). Process of the webpage of Structs 2.x.

If the framework of Struts2.x and Spring2. $x$ are added, an unclear performance process can be shown as the following Fig. (2).

After users open the page of JSP by browser, enter and submit the formula, a request to server is generated. The server will filter the request, then it will find out whether the Action will be handled and if so, then it will be handled in the configuration file of Struts. Xml. If ID of Action to be performed is found, Spring will find out the corresponding Action according to the configuration of Application Context. Xml, and generate the examples to be input into the place for implementing the Action.

When the Action is finished performing, it will find out the corresponding jump and take the jumping operation in the Struts. xml file. The jumping here can be a page of JSP as well as an Action. If it jumps into the JSP page, we can display the performance results of Action to users.

\subsection{Detailed Design}

The realization of the system function is based on the integration of the framework of Struts2 and Spring, which is developed with layers pattern in accordance with the design model of MVC; the label of Struts2 applied in the presentation layer is responsible for the output of the data and the treatment of the processing logic, and the Action jumping in Struts. Xml; Spring is used to manage the Action/Bean ap- plied in the system and MATLAB is used for value calculation.

The development circumstances of the system is as shown in Table $\mathbf{1}$

The establishment of the circumstance:

Table 1. The development circumstances of the System.

\begin{tabular}{|c|c|}
\hline The name of the Tool (Framework) & Edition \\
\hline \hline $\begin{array}{c}\text { The integration deve-loping circum- } \\
\text { stance of My Eclipse }\end{array}$ & 8.0 \\
\hline Struts2 & 2.0 .1 \\
\hline Spring & 2.0 \\
\hline Tomcat Server & 6.0 \\
\hline JDK & 1.6 \\
\hline MATLAB & $\mathrm{R} 2007 \mathrm{a}$ \\
\hline
\end{tabular}

\section{1) Leading-in Relevant Jar Package}

Leading-in the relevant jar package of Struts2 and Spring2, leading-in the Javabuilder. jar in MATLAB, and 
leading-in the numerical function jar package of MATLAB. The jar package of MATLAB function will be shown detailed in the key link.

\section{2) The Configuration of the Profile}

Web.xml: the initialized configuration information. Filter, filter-mapping, listener are configured in the profile, which also can be configured by welcome-file-list and defaulted as the display page.

An example of configuration is as follows:

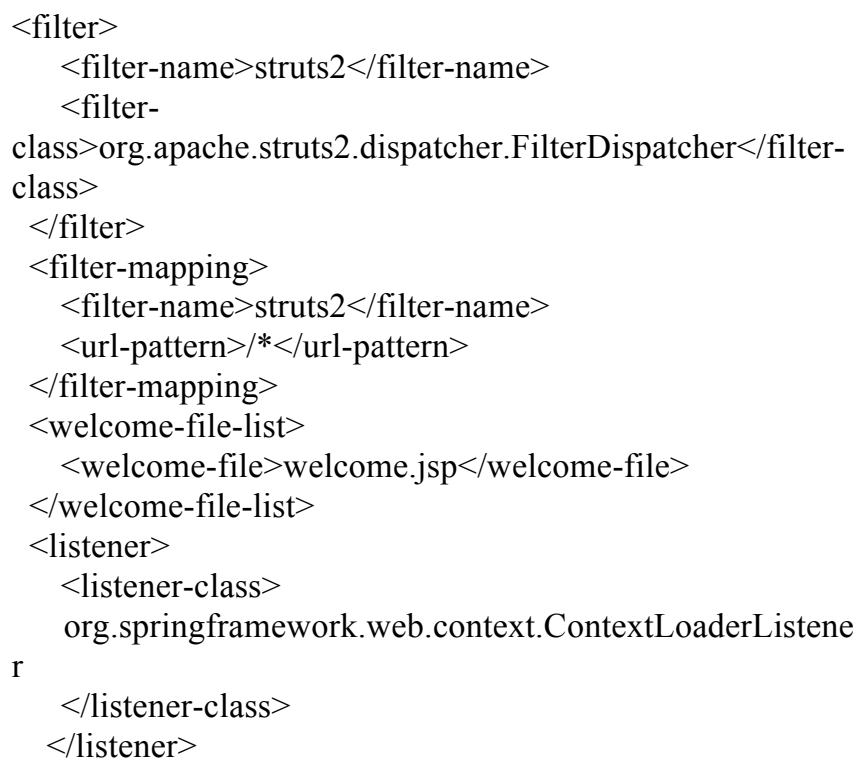

(1) Struts.xml, which is a default configuration file of Struts2. It has configured the interceptor, Action, the correspondence between the Action and the user requests, the jumping after the performance of Action, and the interceptor applied for different users.

An example of configuration is as follows:

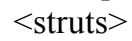

Application context.xml, which is the default configuration file for Spring. The Action case in Struts 2 can be managed and generated by Spring.

$$
\begin{aligned}
& <\text { bean id="computeFormula" } \\
& \text { class }=" \text { cugb.action.computeFormulaAction" } \\
& \text { scope }=\text { "prototype" }> \\
& <\text { property } \\
& \text { ref="analyzeFormula" }>> \\
& <\text { property } \\
& \text { name="analyzeFormula" } \\
& \text { name="useMATLAB" } \\
& \text { ref="useMATLAB"/> } \\
& </ \text { bean }>
\end{aligned}
$$

\section{3) JSP page}

A form is mainly required on the JSP page, which submits the mathematical formulas input by users onto the server for processing. We can apply the labels provided by Struts 2 to add form on the JSP page. Before applying the form of Struts2, we need to introduce the label of Struts2. And then the following line of code shall be added in front of the JSP page.

$<\% @$ taglib prefix="s" uri="/struts-tags $\% \%>$

Then, the label of struts 2 can be applied on the JSP page, which is as follows.

$<$ s:form action="computeFormula.action" $>$ $<$ s:textfield name="formula" label=" Please enter

the formula $">>$

$$
<\text { s:submit label=" Submit "/> }
$$$$
</ \mathrm{s} \text { :form }>
$$

\section{4) Action}

In the Action of Struts2, it is easy for us to receive the data sent by users. We just need to definevariables in the Action files and its method of obtaining and setting, then, the variables can be directly used to execute the method, which has been assigned the data input by users. It is certainly noted that the variable name defined and the property of the member name in the form of the JSP page are consistent. If the name property in the $<$ s:textfield $/>$ of the JSP page is formula, then we shall define a variable called formula in computeFormulaAction.action. The execute() in Action shall succeed the ActionSupport.

An example of codes is as follows

private String formula;

public String getFormula() \{ return formula;

\}

public void setFormula(String formula) \{ this.formula = formula;

\}

public String execute() throws Exception \{ System.out.println(formula); return $S U C C E S S$; \}

We can extract the analysis and calculation of the mathematical formula and make it a single method, and then we transfer it in computeFormula-

Action.action,. When we apply these method in computeFormulaAction.action, the Spring can be the dependency injection. In this way, when our analysis and calculation have come to be realized in different ways, only a change of the configuration in the file of applicationContext.xml can realize the replacement of the analysis and calculation without altering the Action files. We just define the method applied by us in the file of compute FormulaAction.action, and their way of obtaining and setting, then, configure it in the configuration file of applicationContext.xml, after that we can use these methods in the excute (). 


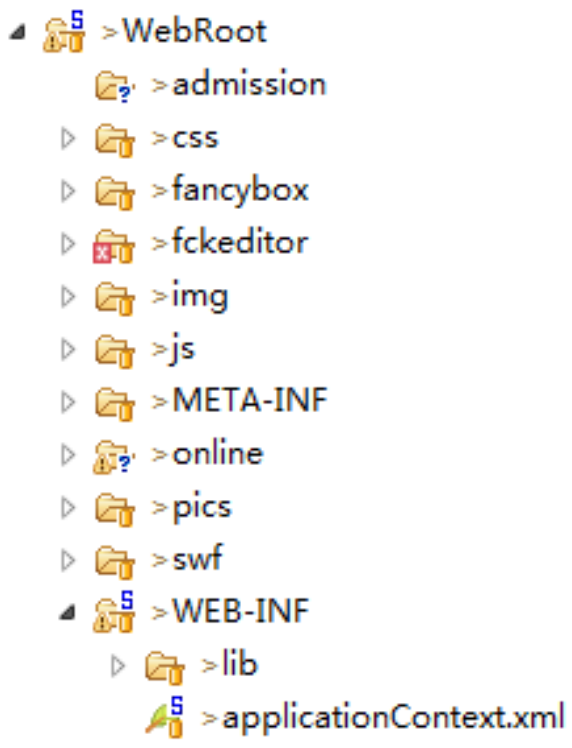

Fig. (3). The MATLAB function in the formula. jar in Java.

\section{THE KEY LINK}

The key in the system lies in how to formulate the Jar package of MATLAB function, the MATLAB function transferred in Java, and the analysis and decomposition of the mathematical formula. Here we outline the first two briefly.

\subsection{Self-Making the Jar Package of the MATLAB Func- tion}

Take the MATLAB r2007a as an example.

Firstly, circumstance settings are implemented. MATLAB r2007a, JDK1.6 is provided in computer, and the circumstance variable, JAVA HOME, is set to arrange the path for JDK; the path of matlabInstallRoot

\toolbox \javabuilder\jar\javabuilder.jar

is added in the classpath, the path of

\section{\%JAVA_HOME\%/bin/javac}

is added into Path.

Then, the software of MATLAB r2007a is executed, inputting the command of deploytool in the command window, a window of deploytool will pop up, clicking the New button, selecting the Matlab Builder for Java and Java Package. Createing a new project of formula,prj. It is ensured that the Generate Verbose Output has been checked in the Deployment Tool Panel.

Secondly, the $\mathrm{m}$ file to be transferred by Java (such as mydraw.m, including two parameters $(\mathrm{x}, \mathrm{y})$ ), will be dragged and from the left interface panel of the Matlab working di- rectory to the class folder newly built in the Deployment Tool.

Finally, click the build button, then a folder with a same name as the project name is generated under the current directory of Matlab. Formula.jar will be found in the folder, which is The Jar package of MATLAB function that we need.

\subsection{The Application of MATLAB Function in Java}

Open the development tool of My Eclipse, add the two jar package, ie. formula.jar, javabuilder.-jar into the lib folder under the WEB-INF folder in the WebRoot of the project. (Fig. 3).

Then, we can use the MATLAB function in the formula.jar in Java.

\section{CONCLUSION}

Based on the existing project basis of Java EE, the system has introduced the jar package containing the MATLAB function, which helps us introduce the MATLAB function for the value calculation of the mathematical formula, which requires that the users shall strictly comply with the mathematical formula of Latex when inputting the formula.

\section{CONFLICT OF INTEREST}

The author confirms that this article content has no conflict of interest.

\section{ACKNOWLEDGEMENTS}

This paper belongs to the project of the "A Project of Shandong Province Higher Educational Science and Technology Program (J14LI57)", "Teaching reform project of Shandong Jiaotong University (JG201434)", "Scientific Research Foundation of Shandong Jiaotong University (Z201428)" and "Dr. Startup funds of Shandong Jiaotong University".

\section{REFERENCES}

[1] D. E. Knuth, The TEXbook. Menlo Park: Addison Wesley, cop. 1996.

[2] C. Jie, MATLAB Collection (2 ${ }^{\text {nd }}$ ed.), 2010.

[3] X. Dingye, and C. Yangquan, Advanced Applied Mathematical Problem Solution with MATLAB (2 ${ }^{\text {nd }}$ ed.) Tsinghua University Press, 2008.

[4] E. Jendrock, J. Ball, D. Carson, I. Evans, S. Fordin, and K. Haase, The Java EE 5 Tutorial (3 ${ }^{\text {rd }}$ ed.). Prentice Hall: USA, 2008.

[5] L. Gang, Struts 2.1. Tutorial, 2009.

[6] L. Xiaohua, Z. Jian, and Z. Huizhen, JSP Application Developing Detail Explain (3 ${ }^{\text {rd }}$ ed.). 2007.

[7] C. Wall, and R. Bredenbach, Spring in Action: Manning. 2008.

Received: November 26, 2014 